\title{
On Cycles in AS Relationships
}

\author{
Xenofontas \\ Dimitropoulos \\ IBM Research, Zürich \\ xed@zurich.ibm.com
}

\author{
M. Ángeles Serrano \\ IFISC (CSIC-UIB) \\ marian.serrano@ifisc.uib- \\ csic.es
}

\author{
Dmitri Krioukov \\ CAIDA \\ dima@caida.org
}

This article is an editorial note submitted to CCR. It has NOT been peer reviewed. Authors take full responsibility for this article's technical content. Comments can be posted through CCR Online.

\section{Categories and Subject Descriptors \\ C.2.5 [Local and Wide-Area Networks]: Internet; C.2.1 [Network Architecture and Design]: Network topology}

\section{General Terms}

Measurement, Design, Verification

\section{Keywords}

AS relationships, cycles

$\mathbf{S}$ leased with [2], asked us why it contained AS relationship cycles, e.g., cases where AS $A$ is a provider of AS $B, B$ is a provider of $C$, and $C$ is a provider of $A$, or other cycle types. Having been answering these questions in private communications, we have eventually decided to write down our answers here for future reference.

Formally, the heuristics in [2] do not produce acyclic relationships because neither do the techniques that [2] is based on [3, 4, 5], and because we did not try to impose any no-cycle constraints that can certainly be enforced [6]. Below we explain why we did not try to do that, and thus behaved somewhat counter-intuitive.

First, we have to emphasize that AS relationship cycles by no means imply or induce routing or traffic forwarding loops, since the BGP's routing loop avoidance mechanism, i.e., AS path checking, does not depend on any AS relationships. Another problem with BGP causes some confusion sometimes. BGP can diverge due to policy conflicts [7], where policy refers to a ranking of prefixes that can be used to reach a destination. As AS relationships determine export policies, they may also influence prefix rankings, e.g., an AS typically prefers to reach a destination via its customer, then peer, then provider. However, this influence by no means leads to BGP divergence, which is a separate problem with BGP that can only appear in the presence of non-shortest-path routing and conflicting path selection policies. AS relationship cycles present or not, the default BGP path selection mechanism that chooses shortest paths, or many other possible safe rankings [7] result in stable and loopfree routing.

Second, we have never received a satisfactory answer to our counter question: Why can the global Internet not have any AS relationship cycles? Why does it have to be a DAG (directed acyclic graph)? AS relationships emerge from business negotiations between pairs of ASs, while all relevant information is kept secret. In other words, local negotiations and interactions between AS pairs determine their relationships. Given the complexity of business agreements, it is quite unlikely that the local, independent, and diverse interactions between ASs yield a global, highly organized, and strictly hierarchical DAG structure. What entity would enforce and control this structure? Perhaps this entity is "rational economy," in which there is no money transfer cycles? In stricter terms, the question is if the Internet market complies with the efficient market hypothesis [8]. We emphasize that there is no data to answer this question either way. We believe it is unlikely that the Internet is a perfectly efficient global market, and refer an interested reader to the criticism of the efficient market hypothesis in [9].

Another reason why AS relationship cycles may exist in the Internet is that real AS relationships, especially those between large ISPs, may be more complex than the course modeling abstraction we adopted in [2]. Real AS relationships may depend on a peering point, prefix, and even time [2]. For example, ISPs that dominate one geographical region can provide transit for this region and receive transit for other regions, thus forming partial transit relationships with other ISPs. Such geographical interdependencies clearly lead to relationship cycles: three ASs active primarily in the US, Europe, and Asia, but also present in the two other regions, may form a cycle by providing transit to each other for their primary regions. Interestingly, this kind of information-losing abstractions of different-type objects or relationships as nodes or links of the same type explain cycles in other flow networks. For example, in the economic network of the world trade web [10], cycles are present due to coarse categorizations of products and contractual relationships. Food webs (nodes are species and directed links show who eats whom) also have cycles [11], quite a counter-intuitive fact if one considers the biomass flux, but it is easily explainable by heterogeneities in population composition such as size, age, etc. Going back to the Internet, the diversity of real business agreements between ASs is a sufficient condition for the presence of cycles.

Finally, we did not claim the $100 \%$ accuracy of our results. Our validation indicated that they were roughly $90 \%$ accurate [2]. Incorrect inferences are thus present in [1]. Suppose that our heuristics mis-infer a single relationship between a small customer AS $C_{1}$ and its provider, large ISP $P$. A single erroneous relationship of this type can result in many cycles, as all other customers $C_{i}$, $i=2,3, \ldots$, of $P$ will then have a path to $C_{1}$ in the customerto-provider direction. Given that $C_{1}$ can also have other customerto-provider paths to $C_{i}$ via its providers other than $P$, this type of mis-inference will form many cycles going via $P, C_{1}$, and $C_{i}$. Clearly, no-cycle constraints should in theory suppress this type of errors; we also considered making the minimization of the number of cycles an objective in our multiobjective problem formulation, but given all the points above suggesting that AS relationship cycles may in fact be present in the real Internet, we decided not to follow this path. 
[1] CAIDA. AS Relationships Data. Research Project. http://www.caida.org/data/active/as-relationships/.

[2] X. Dimitropoulos, D. Krioukov, M. Fomenkov, B. Huffaker, Y. Hyun, kc claffy, and G. Riley. AS relationships: Inference and validation. Comput Commun Rev, 37(1), 2007.

[3] X. Dimitropoulos, D. Krioukov, B. Huffaker, kc claffy, and G. Riley. Inferring AS relationships: Dead end or lively beginning? In WEA, 2005.

[4] G. D. Battista, T. Erlebach, A. Hall, M. Patrignani, M. Pizzonia, and T. Schank. Computing the types of the relationships between Autonomous Systems. IEEE ACM T Network, 15(2), 2007.

[5] L. Subramanian, S. Agarwal, J. Rexford, and R. H. Katz. Characterizing the Internet hierarchy from multiple vantage points. In INFOCOM, 2002.

[6] R. Cohen and D. Raz. Acyclic type of relationships between Autonomous Systems. In INFOCOM, 2007.
[7] T. Griffin, F. B. Shepherd, and G. Wilfong. The stable paths problem and interdomain routing. IEEE ACM T Network, 10(2), 2002.

[8] E. Fama. Efficient capital markets: A review of theory and empirical work. $J$ Financ, 25(2):383-417, 1970.

[9] S. J. Grossman and J. E. Stiglitz. On the impossibility of informationally efficient markets. Am Econ Rev, 70(3):393-408, 1980.

[10] M. Á. Serrano, M. Boguñá, and A. Vespignani. Patterns of dominant flows in the world trade web. J Econ Interact Coord, 2(2):111-124, 2007.

[11] S. Allesina, A. Bodini, and C. Bondavalli. Ecological subsystems via graph theory: The role of strongly connected components. OIKOS, 110(1):164-176, 2005. 